\title{
Robot-assisted partial nephrectomy of initial cases using a 3D square-block type kidney model
}

\author{
Masahiro Yamazaki ${ }^{1}$, Tatsuya Takayama ${ }^{1}$, Akira Fujisaki ${ }^{1}$, Tomoki Kamimura ${ }^{2}$, Toshihiro Mashiko ${ }^{3}$, \\ Tetsuya Fujimura ${ }^{1}$
}

${ }^{1}$ Department of Urology, ${ }^{2}$ Department of Radiology, ${ }^{3}$ Department of Neurosurgery, Jichi Medical University Hospital, Tochigi, Japan

Contributions: (I) Conception and design: M Yamazaki, T Takayama; (II) Administrative support: T Takayama, T Mashiko, T Fujimura; (III) Provision of study materials or patients: M Yamazaki, T Takayama, A Fujisaki, T Kamimura, T Mashiko; (IV) Collection and assembly of data: M Yamazaki, T Takayama, A Fujisaki, T Kamimura, T Mashiko; (V) Data analysis and interpretation: All authors; (VI) Manuscript writing: All authors; (VII) Final approval of manuscript: All authors.

Correspondence to: Tatsuya Takayama, MD, PhD. Department of Urology, Jichi Medical University, 3311-1 Yakushiji, Shimotuke city, Tochigi 3290498, Japan. Email: ttakayam@jichi.ac.jp.

\begin{abstract}
Background: It is extremely important to understand the local anatomy prior to performing appropriate and efficient robot-assisted partial nephrectomies (RAPNs).

Methods: We developed a personalized three-dimensional printed kidney model of square-block type to enhance our knowledge and understanding on the underlying anatomy during RAPN, and we consequently applied this model to six initial cases of RAPN.

Results: The mean warm ischemic time was 18 minutes and the mean estimated blood loss was $59 \mathrm{~mL}$. Only one patient presented with a minor complication, whereas all six patients included in this study were surgical margin negative.

Conclusions: We believe that this cost-effective model helped us in understanding the underlying local anatomy and facilitating an increased efficiency in the related surgery. Further studies are required to validate our preliminary results.
\end{abstract}

Keywords: Partial nephrectomy (PN); three-dimensional (3D); kidney model; square, economical

Submitted Sep 18, 2019. Accepted for publication Dec 09, 2019.

doi: $10.21037 /$ tau.2019.12.31

View this article at: http://dx.doi.org/10.21037/tau.2019.12.31

\section{Introduction}

The use of ultrasonography and cross-sectional imaging has now facilitated a distinct increase in the incidental detection of small renal masses (SRMs) (1). On the other hand, partial nephrectomy $(\mathrm{PN})$ is a standard treatment for SRMs (1). Therefore, due to the widespread use of robotic surgery for a broad range of procedures, robot-assisted PN (RAPN) is rapidly becoming a predominant method for treating SRMs

In a previously published report pertaining to the learning curve of RAPN, the authors stated that in ten cases examined the warm ischemic time (WIT) was found to be 20 minutes, whereas the probability of Clavien-Dindo $\geq 2$ complication-free postoperative course was $77 \%$ (2). Hence, in order to obtain better results in initial cases, it becomes imperative to emphasize on the respective anatomical understanding and on performing efficient and appropriate surgical planning processes.

Renal tumor models developed by three-dimensional (3D) printing have been reported for facilitating efficient preoperative planning in PN (3). More specifically, these 3D models were produced by the use of translucent resin, silicon, etc. (4-7). However, in these models, the kidney parenchyma is generated using silicon; therefore, the structure of renal pelvis and blood vessels is difficult to understand.

Therefore, the primary objective of this research study was to create a model that is cost-efficient and 
with anatomical features that are easier to understand. Consequently, we assessed their functionality and efficiency in early cases of RAPN.

\section{Methods}

\section{Patients}

Six patients that had already been diagnosed with renal cell carcinoma (cT1aN0M0) underwent RAPN at our hospital between March 2016 and December 2016. The mean age of these patients was 54 years (range, 37-67 years). Following approval from the Institutional Review Board of Jichi Medical University (No. B15-068), we developed $3 \mathrm{D}$-printed models for these six patients prior to their surgical processes.

\section{Surgeon background}

A single surgeon without extensive experience in RAPN performed the surgical processes in all patients included in this study. The surgeon has been working in the urology department for the last 24 years and he has performed more than 50 open PN, 40 robot-assisted radical prostatectomies but only 3 RAPNs. Furthermore, he had no previous experience with laparoscopic PN.

\section{RAPN procedure}

These cases of RAPN were performed using the da Vinci Si Surgical system (Intuitive Surgical, Sunnyvale, California, USA). In all cases, a transperitoneal approach of RAPN with early unclamping technique was employed. It should be also mentioned that we performed an early unclamping technique, in which the arterial clamp was released after the running sutures on the tumor bed (8).

\section{Development of the 3D model}

First, patient contrast-enhanced computed tomography (CT) images of were obtained and the CT protocol involved the SOMATOM Definition FLASH CT (Siemens Healthineers, Munich, Germany; slice thickness, $0.75 \mathrm{~mm}$ ). A contrast medium (350 mg iohexol) was injected at $540 \mathrm{~mL} / \mathrm{kg}$ over a time period of 25 seconds.

Secondly, the acquired digital images were visualized in three dimensions using Ziostation2 (Ziosoft, Tokyo, Japan) and SYNAPSE Vincent (Fuji Film, Tokyo, Japan).
All 3D images depicting the kidney outline, the urinary tract, the blood vessels, and the tumor were saved separately as Digital Imaging and Communication in Medicine data. Consequently, they were converted to Standard Triangulated Language files using Osirix (Pixmeo SARL, Geneva, Switzerland) $(9,10)$, and then synthesized using a Meshmixer (Autodesk, California, USA). A Meshmixer was also used to reproduce the outline of the kidney in a grid.

Third, a UP Plus2 (Beijing Tiertime Technology Co., Ltd., Beijing, China) 3D printer was used to generate a $3 \mathrm{D}$ model from these files. This $3 \mathrm{D}$ printer injects acrylonitrile butadiene styrene (ABS) resin as the printing material from a nozzle to repeatedly draw $0.15-\mathrm{mm}$ thick pattern layers on a platform at $260{ }^{\circ} \mathrm{C}(9)$. Model printing with the UP Plus2 commenced following trimming of the $3 \mathrm{D}$ data within the necessary range for anatomical understanding (Figure 1A). Eventually, the model was printed life-size and all unnecessary parts such as the pedestal and support are detached by pliers, whereas all remaining parts are painted (Figure $1 B, C, D, E$ ). Finally, the three parts are bonded with a soldering iron to complete the model (Figure $1 F, G, H$ ). The models were used for preoperative planning and for verifying the anatomical structures during surgery.

\section{Results}

Half of the patients' tumor lesions were at the right-hand side and half were on the left one. The mean tumor size was $19 \mathrm{~mm}$ (range, $13-32 \mathrm{~mm}$ ), and the mean R.E.N.A.L nephrometry score was 6.7 point (range, 5-8 point) (11) (Table 1). An example of enhanced CT image and its subsequently created 3D models are shown. The models managed to illustrate the 3D image in detail (Figures 2-5), and hence they were able to reproduce the anatomical structure explicitly compared to the actual intraoperative image (Figure 6).

Table 2 depicts the clinical results of the six patients included in this study. The mean WIT and console time and estimated blood loss (EBL) were 18.0 minutes (range, 12.031.2 minutes), 167 minutes (range, 89-250 minutes) and $59 \mathrm{~mL}$ (range, 5-100 mL), respectively. In all cases, there were no intraoperative complications identified. A complication was observed in one patient and which involved the development of a 5-mm-sized pseudoaneurysm following 1 week from the RAPN process. However, enhanced CT confirmed that it eventually disappeared without intervention following one month and with no recurrence. Furthermore, there perioperative blood 

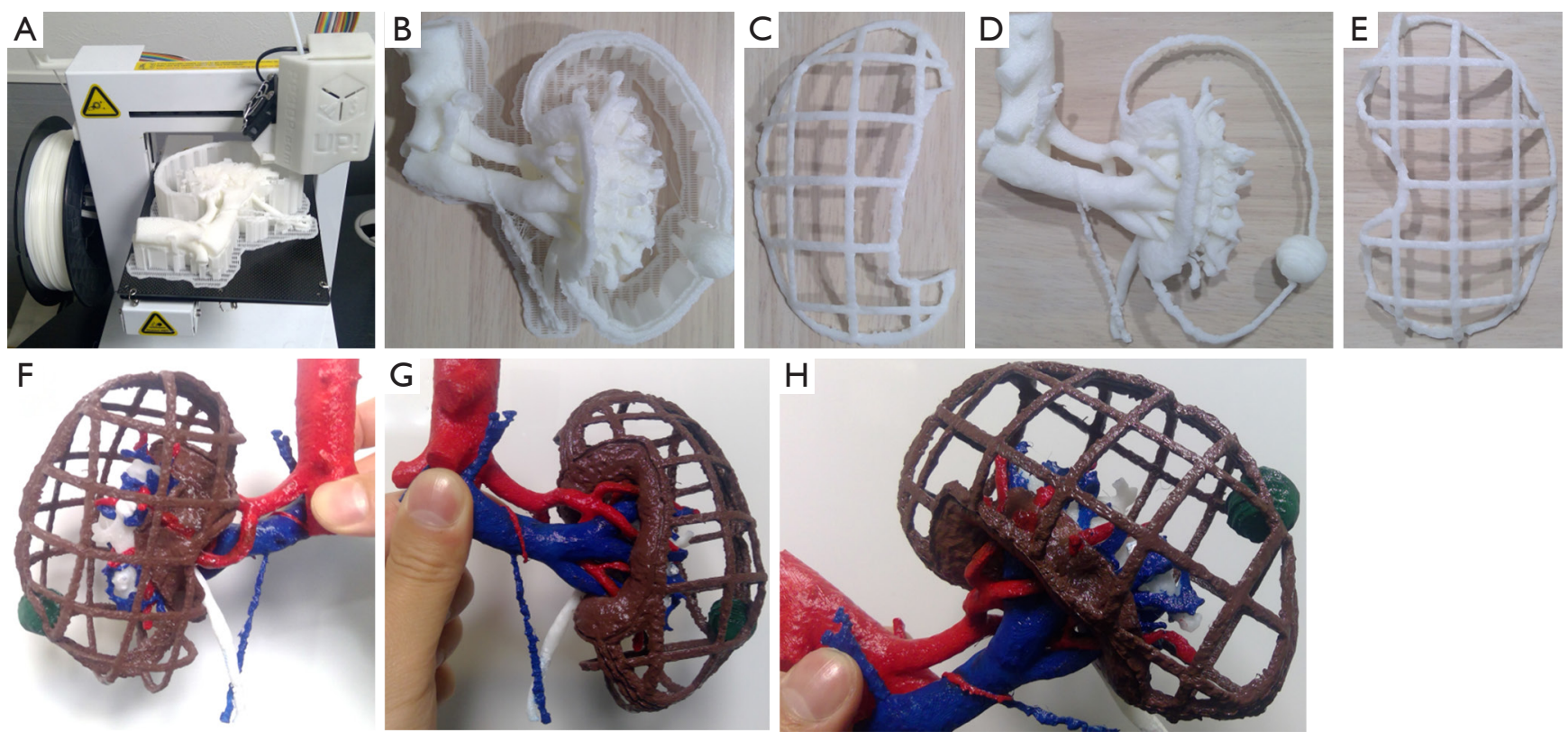

Figure 1 The model used for case 3 patient. 3D model of a left renal tumor with a R.E.N.A.L nephrometry score of 5p point (R1E2N1AxL1). $(A, B)$ Initial 3D model based on the three-dimensional image; (C,D,E) the unnecessary parts were detached from "B"; (F,G,H) green, brown, red, blue, or white color indicate renal tumor kidney, artery, vein or correcting system, respectively. The 3D model can be easily rotated in order to evaluate the precise relationship between renal tumor and feeding vessels or renal pelvis. 3D, three dimensional.

Table 1 Clinical characteristics of the six patients with RAPN

\begin{tabular}{|c|c|c|c|c|c|c|}
\hline Characteristics & Case 1 & Case 2 & Case 3 & Case 4 & Case 5 & Case 6 \\
\hline Sex & Female & Male & Male & Female & Female & Male \\
\hline ASA & 1 & 1 & 2 & 1 & 2 & 2 \\
\hline Tumor site & Right & Left & Left & Left & Right & Right \\
\hline Tumor size $(\mathrm{mm})$ & 14 & 21 & 18 & 19 & 32 & 12 \\
\hline R.E.N.A.L score & $1-3-1-a-1$ & $1-1-3-a-1$ & $1-2-1-x-1$ & $1-3-1-p-3$ & $1-1-3-p-2$ & $1-3-2-x-2$ \\
\hline Preoperative eGFR $\left(\mathrm{mL} / \mathrm{min} / 1.73 \mathrm{~m}^{2}\right)$ & 86 & 77 & 87 & 92 & 96 & 62 \\
\hline
\end{tabular}

RAPN, robot-assisted partial nephrectomy; ASA, American Society of Anesthesiologists physical status classification; eGFR, estimated glomerular filtration rate.

transfusion was not used and there was no need to employ an open surgery. All six patients did not present with recurrence tumors following a median duration of 30 months post-surgery (range, 28-36 months). Finally, and with respect to the generation of the $3 \mathrm{D}$-model, mean time was 17 hours and the cost was USD \$10 per case.

\section{Discussion}

We performed six cases of RAPN using a $3 \mathrm{D}$ squareblock type kidney model. This initial experience with RAPN revealed efficient results in all but one case, and the development of the 3D models was cost-effective. To our best knowledge, there is only one published case report that 

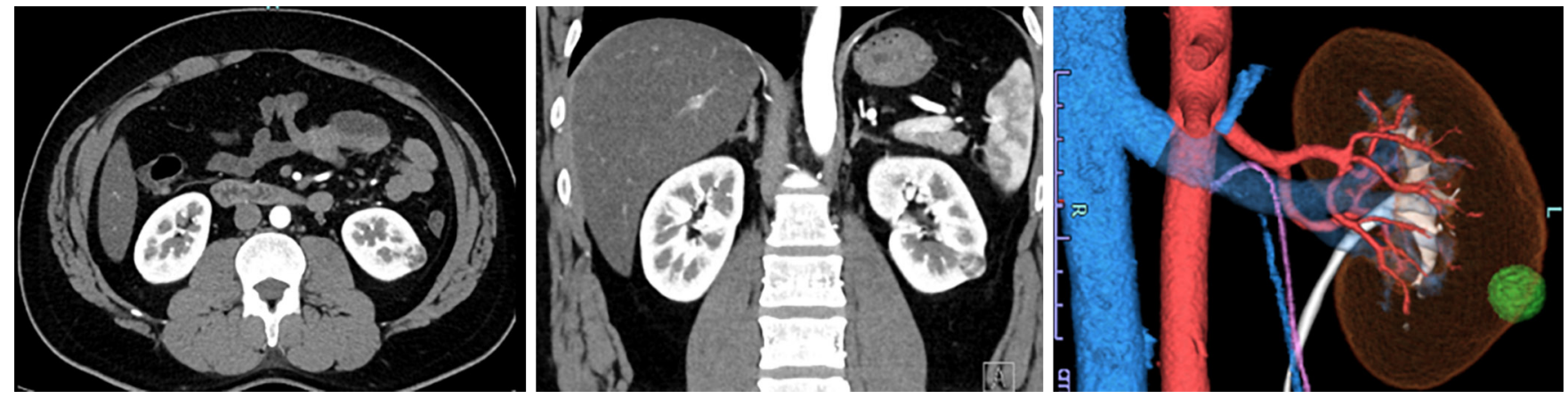

Figure 2 This is the enhanced CT for case 3 patient, depicting coronal, sagittal, and 3D images. CT, computed tomography; 3D, three dimensional.
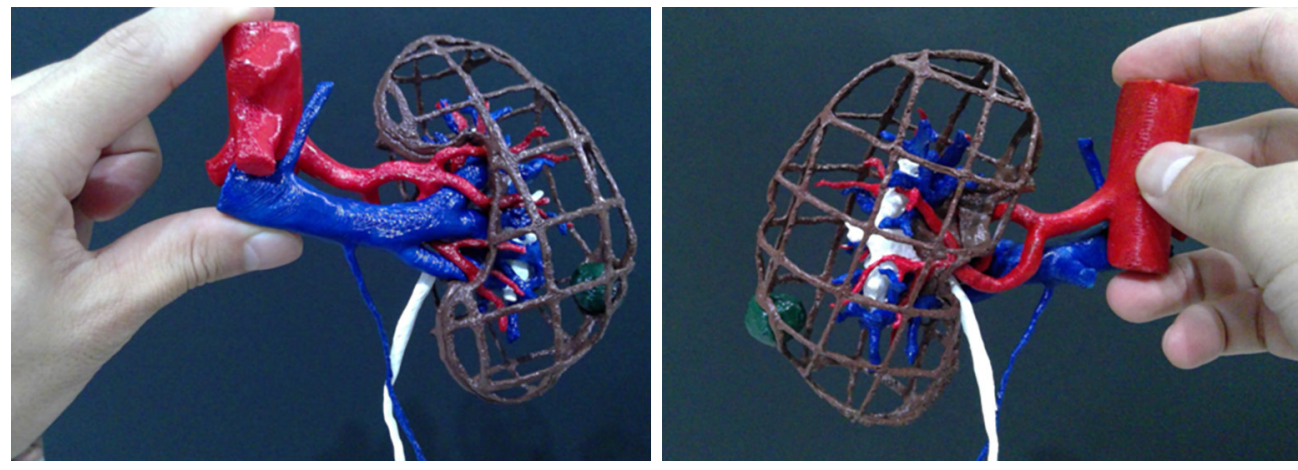

Figure 3 3D model of case 3 patient, showing anterior and posterior images. 3D, three dimensional.
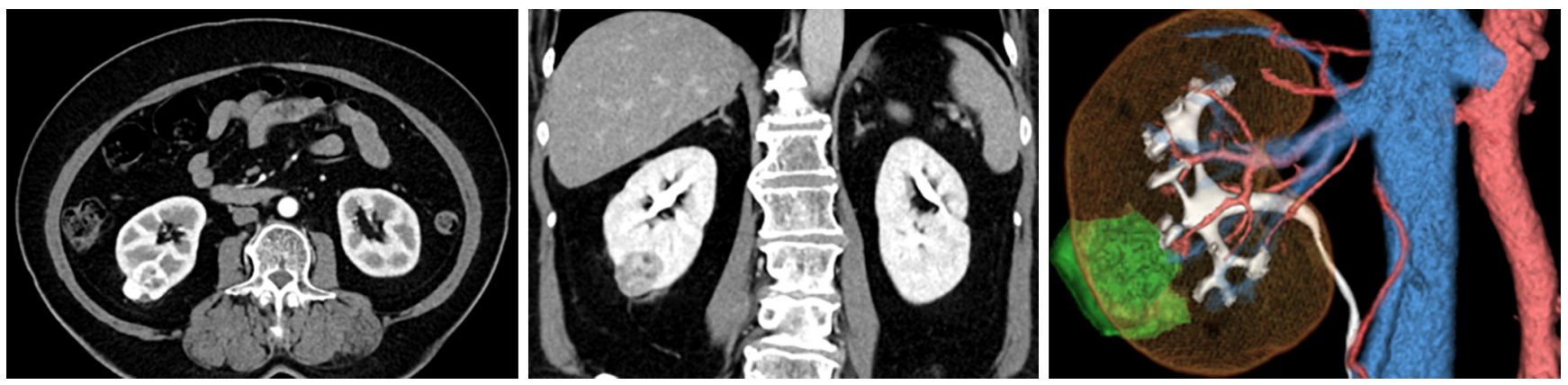

Figure 4 This is the enhanced CT for case 5 patient, depicting coronal, sagittal, and 3D images. CT, computed tomography; 3D, three dimensional.

describes initial cases of RAPN with square-block type 3D model.

Compared to previous reports pertaining to initial experiences with RAPN, our case studies exhibited very promising and efficient results (12). In these reports, the mean WIT was over 20 minutes and the mean EBL were over $90 \mathrm{~mL}$. Evidently, there are differences approaches employed during the surgical process, as we performed RAPN using a technique of early unclamping, in order to decrease the respective WIT (8). Although this 

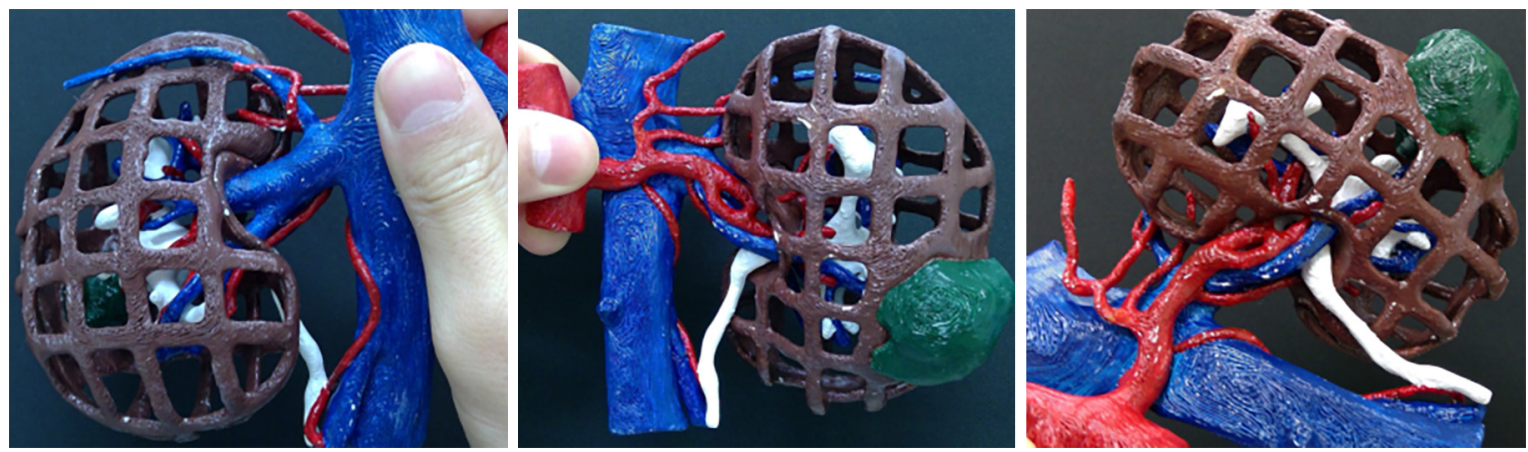

Figure $53 \mathrm{D}$ model of case 5 patient, showing anterior and posterior images. The third image shows that renal pelvis and tumor are close to each other. $3 \mathrm{D}$, three dimensional.
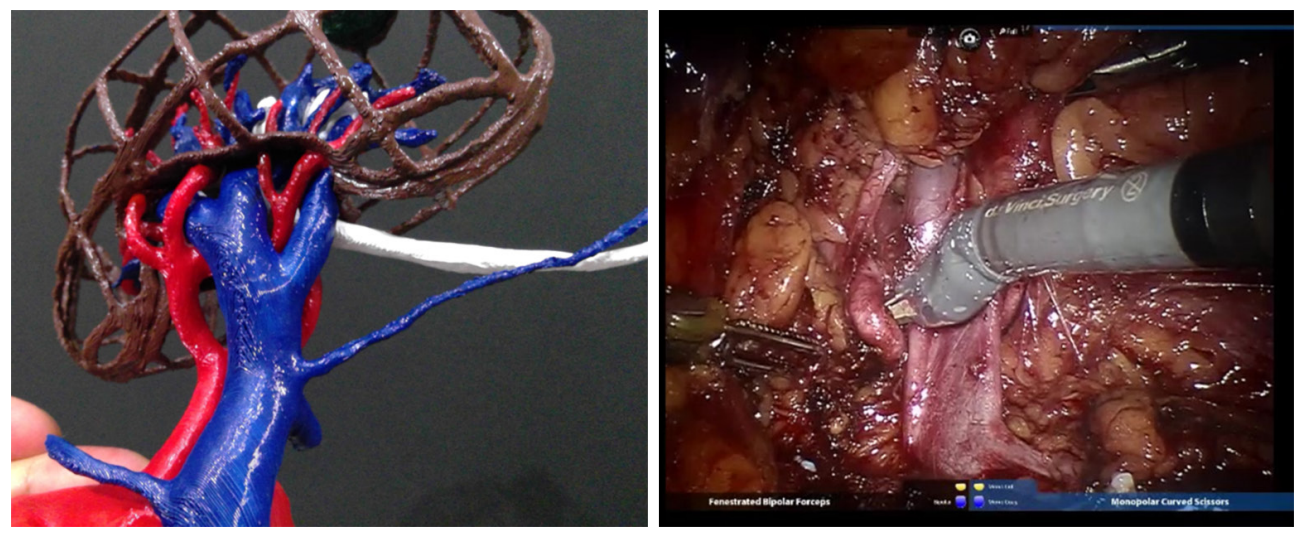

Figure 6 Comparison between the model and the actual surgical image. The model reproduces the anatomical structure in detail.

Table 2 Pathological and surgical results of the six patients

\begin{tabular}{|c|c|c|c|c|c|c|}
\hline Variables & Case 1 & Case 2 & Case 3 & Case 4 & Case 5 & Case 6 \\
\hline Estimated blood loss (mL) & 5 & 100 & 50 & 50 & 100 & 50 \\
\hline Resected tumor volume (g) & 1.0 & 12.9 & 3.9 & 1.9 & 21.0 & 5.6 \\
\hline Surgical margin & Negative & Negative & Negative & Negative & Negative & Negative \\
\hline Console time (minutes) & 89 & 204 & 175 & 176 & 250 & 105 \\
\hline $\begin{array}{l}\text { Postoperative eGFR three months after surgery } \\
\left(\mathrm{mL} / \mathrm{min} / 1.73 \mathrm{~m}^{2}\right)\end{array}$ & 81 & 83 & 100 & 73 & 89 & 60 \\
\hline
\end{tabular}

Clear cell, clear cell renal cell carcinoma; papillary, papillary renal cell carcinoma; eGFR, estimated glomerular filtration rate. 
technique was performed by low-experienced surgeons ( $<20$ procedures), the mean WIT was 17.2 minutes and the mean EBL was $320 \mathrm{~mL}$, whereas the complications rate was $25.8 \%$ (8). Therefore, the significance of our findings is that our results were lower levels EBL and complication rates compared to other published studies.

In recent years, several reports have highlighted the usefulness of 3D modeling for RAPN. The 3D model was anatomically accurate in reports. The surgical procedure can be improved because it is easy to use this model for surgical planning $(13,14)$.

However, it has been reported that although a $3 \mathrm{D}$ model is used, some cases can have a positive surgical margin for the tumor. No reports exist in which the renal parenchyma is clearly observed. Thus, our model that can adequately depict the renal parenchyma and provide with a greater anatomical understand regarding the tumor's depth is bound to be extremely useful.

Our findings suggest that our square type model allowed us to better understand the detailed positional relationship between the arteries and veins involved, and hence we could clamp the artery we wanted. In hepatectomy, there are reports that a 3D-printed frame model was helpful for recognizing the spatial relationship among the tumor, blood vessels and the liver $(15,16)$. An advantage in the model is that because of there are no light reflection from the material, an outer frame model was useful for confirming the anatomical structure $(15,16)$. It should be stated that our model cannot be used for training because various types of models are already reported (17).

Moreover, the development of our own 3D models was relatively cost-effective. Systematic reviews have underlined that the cost of a $3 \mathrm{D}$ model is between USD $\$ 1$ and USD $\$ 1,000$, whereas the majority of these models will cost more than USD \$100. According to two systematic reviews, the cost of making such models by country is $\$ 3.9-1,000$ in North America (4 reports), \$7.4-450 in Europe (5 reports), $\$ 1$ or 200 in Oceania (1 report), \$100-680 in Asia (three reports). Hence, and considering these data, our model was generated at a relatively low cost $(18,19)$.

Nevertheless, we initially tried to develop a model in which the renal parenchyma could be reproduced with a crystal resin, however, the related cost was approximately USD \$50 per case. Therefore, we develop our models using 3D square-block type kidney model that did not reproduce the parenchyma. Consequently, we were able to reduce the overall cost to USD \$10 per case and, more importantly, the anatomical structure was easier to understand. Naturally, the widespread use of home-made $3 \mathrm{D}$ printers, has triggered the generation of even less expensive cost models (USD \$1) (20). In fact, and in that previous study, the authors demonstrated that their home-made model could accurately demonstrative renal anatomical structures and renal tumors. Therefore, our focus will be on improving our current processes, and hence produce an even less expensive model that retains the same or better quality.

\section{Limitations}

There are several limitations in our study design. For instance, we relied on one surgeon to perform the operations. Therefore, the acquired results may overestimate the efficiency of our design due to the small number of operations performed and because of the absence of highrisk cases. In addition, it was inconclusive whether treatment outcomes would change with or without the $3 \mathrm{D}$ model, or whether using the 3D model decreases the time needed in the respective learning curve. All these issues require additional investigation in future studies that will compare our findings with high score cases or cases without a $3 \mathrm{D}$ printer.

\section{Conclusions}

The use of a 3D square-block type kidney model may improve patient outcomes in initial cases of RAPN, and this model can be generated at a lower cost. We need to conduct further experiments with patients, and hence provide a distinct and statistically sound evaluation of the efficiency of this model in clinical practice.

\section{Acknowledgments}

Funding: None.

\section{Footnote}

Conflicts of Interest: All authors have completed the ICMJE uniform disclosure form (available at http://dx.doi. org/10.21037/tau.2019.12.31). The authors have no conflicts of interest to declare.

Ethical Statement: The authors are accountable for all aspects of the work in ensuring that questions related to the accuracy or integrity of any part of the work are appropriately investigated and resolved. This study was approved by the Institutional Review Board of Jichi Medical 
University (No. B15-068). Written informed consent was obtained from all patients.

Open Access Statement: This is an Open Access article distributed in accordance with the Creative Commons Attribution-NonCommercial-NoDerivs 4.0 International License (CC BY-NC-ND 4.0), which permits the noncommercial replication and distribution of the article with the strict proviso that no changes or edits are made and the original work is properly cited (including links to both the formal publication through the relevant DOI and the license). See: https://creativecommons.org/licenses/by-nc-nd/4.0/.

\section{References}

1. Sanchez A, Feldman AS, Hakimi AA. Current management of small renal masses, including patient selection, Renal Tumor Biopsy, Active Surveillance, and Thermal Ablation. J Clin Oncol 2018;36:3591-600.

2. Larcher A, Muttin F, Peyronnet B, et al. The learning curve for robot-assisted partial nephrectomy: Impact of surgical experience on perioperative outcomes. Eur Urol 2019;75:253-6.

3. Wake N, Rude T, Kang SK, et al. 3D printed renal cancer models derived from MRI data: application in pre-surgical planning. Abdom Radiol (NY) 2017;42:1501-9.

4. Silberstein JL, Maddox MM, Dorsey P, et al. Physical models of renal malignancies using standard crosssectional imaging and 3-dimensional printers: a pilot study. Urology 2014;84:268-72.

5. Knoedler M, Feibus AH, Lange A, et al. Individualized physical 3-dimensional kidney tumor models constructed from 3-dimensional printers result in improved trainee anatomic understanding. Urology 2015;85:1257-61.

6. Smektala T, Golab A, Krolikowski M, et al. Low cost silicone renal replicas for surgical training - technical note. Arch Esp Urol 2016;69:434-6.

7. von Rundstedt FC, Scovell JM, Agrawal S, et al. Utility of patient-specific silicone renal models for planning and rehearsal of complex tumour resections prior to robotassisted laparoscopic partial nephrectomy. BJU Int 2017;119:598-604.

8. Peyronnet B, Baumert H, Mathieu R, et al. Early unclamping technique during robot-assisted laparoscopic partial nephrectomy can minimise warm ischaemia without increasing morbidity. BJU Int 2014;114:741-7.

9. Mashiko T, Konno T, Kaneko N, et al. Training in brain retraction using a self-made three-dimensional model.
World Neurosurg 2015;84:585-90.

10. Mashiko T, Otani K, Kawano R, et al. Development of three-dimensional hollow elastic model for cerebral aneurysm clipping simulation enabling rapid and low cost prototyping. World Neurosurg 2015;83:351-61.

11. Kutikov A, Uzzo RG. The R.E.N.A.L. nephrometry score: a comprehensive standardized system for quantitating renal tumor size, location and depth. J Urol 2009;182:844-53.

12. Mottrie A, De Naeyer G, Schatteman P, et al. Impact of the learning curve on perioperative outcomes in patients who underwent robotic partial nephrectomy for parenchymal renal tumours. Eur Urol 2010;58:127-32.

13. Michiels C, Jambon E, Bernhard JC. Measurement Of The Accuracy Of 3d-Printed Medical Models To Be Used For Robot-Assisted Partial Nephrectomy. AJR Am J Roentgenol 2019;213:626-31.

14. Kyung YS, Kim N, Jeong IG, et al. Application of 3-D printed kidney model in partial nephrectomy for predicting surgical outcomes: A feasibility study. Clin Genitourin Cancer 2019;17:e878-84.

15. Oshiro Y, Ohkohchi N. Three-dimensional liver surgery simulation: computer-assisted surgical planning with threedimensional simulation software and three-dimensional printing. Tissue Eng Part A 2017;23:474-80.

16. Oshiro Y, Mitani J, Okada T, Ohkohchi N. A novel three-dimensional print of liver vessels and tumors in hepatectomy. Surg Today 2017;47:521-4.

17. Smith B, Dasgupta P. 3D printing technology and its role in urological training. World J Urol 2019. [Epub ahead of print].

18. Lupulescu C, Sun Z. A systematic review of the clinical value and applications of three-dimensional printing in renal surgery. J Clin Med 2019. doi: 10.3390/jcm8070990.

19. Sun Z, Liu D. A systematic review of clinical value of three-dimensional printing in renal disease. Quant Imaging Med Surg 2018;8:311-25.

20. Liu D, Sun Z, Chaichana, T, et al. Patient-specific 3D printed models of renal tumours using home-made 3D printer in comparison with commercial $3 \mathrm{D}$ printer. J Med Imaging Health Inform 2018;8:303-8.

Cite this article as: Yamazaki M, Takayama T, Fujisaki A, Kamimura T, Mashiko T, Fujimura T. Robot-assisted partial nephrectomy of initial cases using a 3D square-block type kidney model. Transl Androl Urol 2020;9(2):494-500. doi: 10.21037/tau.2019.12.31 